\title{
Toward efficient modification of large gold nanoparticles with DNA
}

R. Gill*a, K. Göeken ${ }^{\mathrm{a}}$, V. Subramaniam ${ }^{\mathrm{a}, \mathrm{b}}$

${ }^{a}$ Nanobiophysics Group, MIRA Institute of biomedical technology and technical medicine, University of Twente, Enschede, The Netherlands; ${ }^{b}$ FOM Institute AMOLF, Science Park 104, 1098

XG, Amsterdam, The Netherlands; *r.gill@utwente.nl

\begin{abstract}
DNA-coated gold nanoparticles are one of the most researched nano-bio hybrid systems. Traditionally their synthesis has been a long and tedious process, involving slow salt addition and long incubation steps. This stems from the fact that both DNA and gold particles are negatively charged, therefore efficient interaction is possible only at high salt concentration. However, unmodified particles are susceptible to aggregation at high salt concentrations. Most of the recent modification methods involve the use of surfactants or other small molecules to stabilize the nanoparticles against aggregation, enabling faster modification. Here we present our result on an alternative route to reach fast modification in low salt conditions, namely, reduction of the charge of DNA. We will discuss both the use of natural DNA under acidic $\mathrm{pH}$ conditions, and the use of DNA with a cationic, spermine-based "tail" which is commercially available under the name ZNA. Additionally we introduce a characterization method based on ensemble localized surface plasmon resonance measurement (LSPR) which enabled us to extract the kinetics of DNA absorbance without the need for fluorescent tags. Lastly we show that the same ZNA-based modification protocol can be effectively used for silver nanoparticle modification.
\end{abstract}

Keywords: Gold, Nanoparticles. DNA, Plasmon, LSPR

\section{INTRODUCTION}

Noble metal nanoparticles, such as those made of gold or silver, can support localized surface plasmons, which are collective oscillations of conduction electrons. As a results of this interaction, the particles exhibit a resonance in both absorption and scattering, which makes these particles interesting candidates as labels both for in-vitro diagnostics ${ }^{1,2}$ and imaging ${ }^{3,4}$. In many biomedical applications, the particles need to be conjugated with a biological recognition element, such as antibodies or DNA. While antibodies spontaneously adsorb on gold nanoparticles when the $\mathrm{pH}$ of the solution is close to the $\mathrm{pI}$ (isoelectric point) of the antibody (i.e. the total charge on the antibody is approximately neutral) ${ }^{5}$, adsorption of DNA to nanoparticles has traditionally been more difficult. Gold nanoparticles are usually synthesized with a negatively charged capping layer (such as citrate), and therefore are electrostatically repelled from DNA which is also negatively charged. Although high salt concentration can be introduced to minimize the charge repulsion, citrate coated gold nanoparticles are not stable at high salt concentrations. Therefore in the early reports on DNA conjugation to gold nanoparticles, incubation times of $24 \mathrm{~h}^{6}$ or $40 \mathrm{~h}^{7}$ were needed. To reach a high DNA density on the particle surface, high salt concentrations should be gradually introduced, for example by slowly evaporating the solvent. ${ }^{8}$

In the last decade, new faster methods based on stabilizing the uncapped nanoparticles with surfactants were introduced ${ }^{9}$

${ }^{10}$ which reduced the modification time from more than a day to several hours. Very recently, a new, two-step, rapid modification method was introduced, which is based on reduction of the electrostatic repulsion (rather than stabilization with surfactants) by using a buffer at acidic $\mathrm{pH}^{11}$. This method showed quantitative adsorption, and stable particles could be produced without high excess of DNA. However, we have recently showed, that while the method works fine with small, $13 \mathrm{~nm}$ diameter nanoparticles, larger particles (such as 40nm diameter nanoparticles) must be concentrated by 50 100x for stable modification without high excess of DNA ${ }^{12}$.

Looking to push the concept of reducing the electrostatic repulsion in order to allow the fast modification of nanoparticles with DNA, we have recently reported on the fast and stable modification of large gold particles with a polyspermine-terminated DNA commercially known as Zip Nucleic Acid (ZNA) ${ }^{13}$ This polycationic tail reduced the negative charge of the DNA while still allowing for efficient and specific hybridization. ${ }^{14}$ In this manuscript we will 
discuss the advantage of the ZNA method compared to the acidic buffer method based on kinetic measurements of plasmon peak shift, and show proof that this method can also be extended to the modification of silver nanoparticles.

\section{MATERIALS AND METHODS}

\subsection{Materials}

40nm diameter citrate coated gold and silver nanoparticles were purchased from Nanocomposix, San Diego, USA. DNA sequences were purchased from IBA lifesciences, Göttingen, Germany. ZNA modified DNA sequences were purchased from Metabion, Martinsried, Germany.

Table 1: DNA Sequences used

\begin{tabular}{|l|l|}
\hline HS-cap & 5’ HS-AAA AAG GTG GAT AAC GTC TT 3’ \\
\hline ZNA-cap & 5’ ZNA4-TTT TTG GTG GAT AAC GTC TT 3’' \\
\hline
\end{tabular}

Where ZNA4 means the insertion of four repeats of spermine-phosphoramidite which after deprotection result in the following structure:

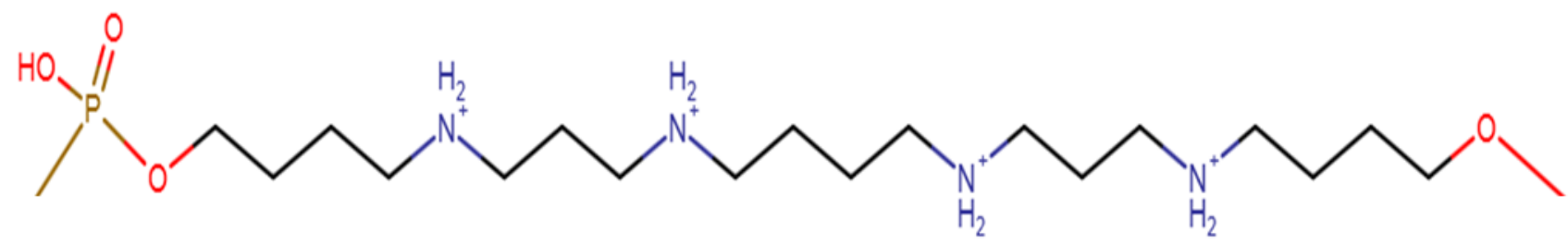

\subsection{Nanoparticle modification with ZNA}

The procedure for modification involved adding $1 \mathrm{~mL}$ of gold nanoparticles (at the stock concentration of the commercial product) to a $1.5 \mathrm{~mL}$ Eppendorf tube, followed by addition of $1 \mu \mathrm{L}$ of $100 \mu \mathrm{M}$ ZNA-modified DNA to the solution, with short vortex mixing. The solution was incubated for 5 minutes at room temperature on the bench. Subsequently, a $200 \mu \mathrm{L}$ aliquot of the solution was transferred to a second Eppendorf, where $90 \mu \mathrm{L} \mathrm{of} 1 \mathrm{M} \mathrm{NaCl}$ solution was added drop by drop on the vortex.

\subsection{Nanoparticle modification with DNA using the acidic citrate method}

The procedure for modification involved adding $1 \mathrm{~mL}$ of gold nanoparticles (at the stock concentration of the commercial product) to a $1.5 \mathrm{~mL}$ Eppendorf tube, followed by addition of $1 \mu \mathrm{L}$ of $100 \mu \mathrm{M}$ DNA to the solution, with short vortex mixing. In a second step, the $\mathrm{pH}$ of the solution was reduced to 3 by addition of $20 \mu \mathrm{l}$ of $500 \mathrm{mM}$ citrate buffer at $\mathrm{pH}=3$. The solution was incubated for 5 minutes (or longer) at room temperature on the bench. Subsequently, a $200 \mu \mathrm{L}$ aliquot of the solution was transferred to a second Eppendorf, where $90 \mu \mathrm{L}$ of $1 \mathrm{M} \mathrm{NaCl}$ solution was added drop by drop on the vortex.

\subsection{Kinetics measurements based on the shift in the peak of the plasmon absorbance band}

Absorbance scans in the range of 500-550 nm, at a step size of $0.2 \mathrm{~nm}$ were performed on a Shimadzu 2401 PC UV-VIS spectrophotometer. The output was saved into an excel sheet. For determination of the peak position, a MATLAB script which reads the excel sheet and finds the peak by fitting the data to a Lorentzian function using the 'lorentzfit' function was used. The 'lorentzfit' function, created by Jered Wells, is freely available on MATLAB central.

\section{RESULTS}

\subsection{Modification of gold nanoparticles - ZNA vs. DNA with acidic citrate buffer}

As a proof of concept of the advantage of using ZNA modified DNA for the fast modification of gold nanoparticles, two Eppendorf tubes with $1 \mathrm{~mL}$ of gold particles were used. One was modified with the ZNA-cap sequence, and the other with the DNA sequence HS-cap, using the protocols mentioned in section 2. As can be seen in Figure 1A, the ZNA modified particles stayed pink in color, which is indicative of the plasmon extinction of unaggregated particles, while the DNA modified particles turned into a dark purple color, which is indicative of coupled plasmons which occurs when 
aggregation takes place. To confirm that we have disperse particles without aggregation when doing the modification with ZNA, we measured the size distribution by dynamic light scattering (DLS), Figure 1B. After the modification of the particles with ZNA, there is a slight increase in hydrodynamic size, without any appearance of a second peak that would indicate the presence of aggregation.

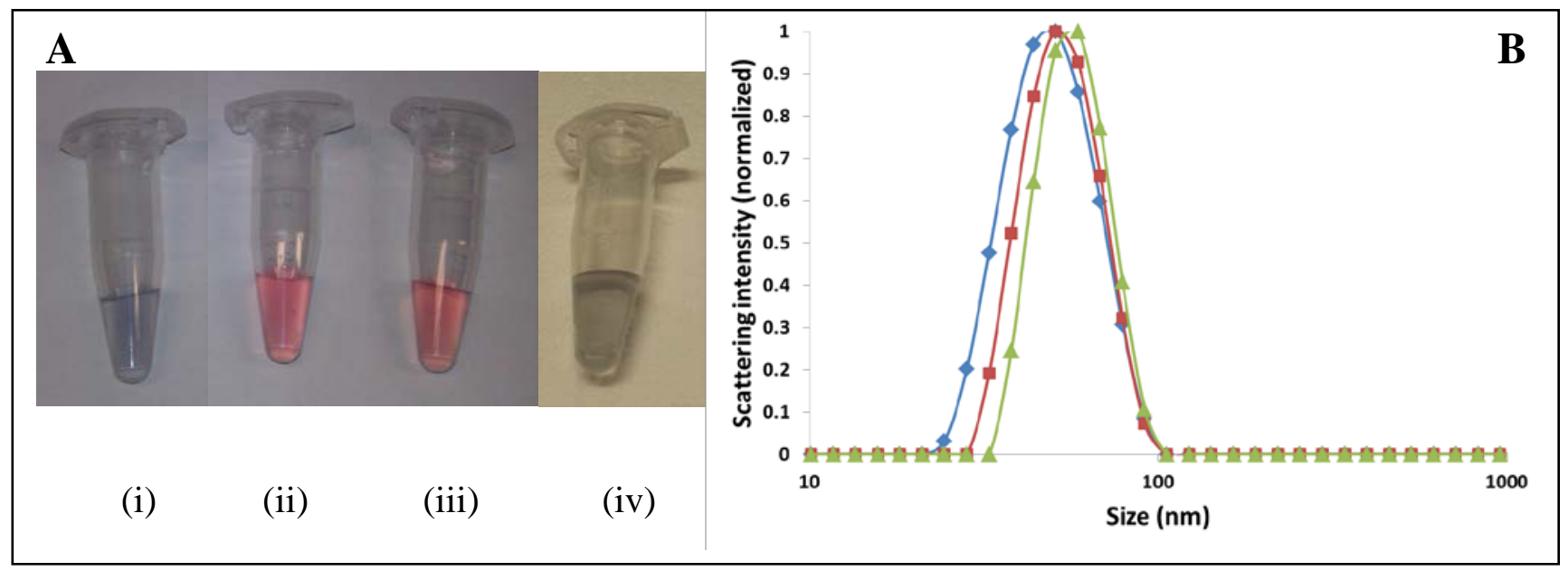

Figure. 1: (A) Pictures of Eppendorf tubes with gold nanoparticles after the addition of $\mathrm{NaCl}$, (i) particles modified with DNA with 5 min incubation at $\mathrm{pH}=3$, (ii) particles modified with $\mathrm{ZNA}$ for $5 \mathrm{~min}$ at $\mathrm{pH}=7$, (iii) unmodified particles diluted in water, (iv) particles modified with DNA with 3 hours incubation at $\mathrm{pH}=3$. (B) DLS measurements of 40nm gold nanoparticles before (diamonds), after 5 minute incubation with ZNA-cap (squares) and after the addition of salt to $\sim 0.3 \mathrm{M}$ final $\mathrm{NaCl}$ concentration (triangles).

\subsection{Kinetics of DNA modification}

We expect that because of its lower total negative charge, the ZNA sequence would bind faster than the DNA sequence, and that could explain why ZNA modified gold particles are more salt stable after 5 minutes of incubation. However, direct observation of the kinetics of attachment of the DNA to the nanoparticles is not a trivial matter. For attachment of DNA to gold surfaces, microgravimetric methods have been reported ${ }^{15}$, but they cannot be used for particles in solution. Several reports exist on following the quenching of dye-labeled DNA to determine the kinetics of attachment, however we have preliminary results that show that the addition of dyes change the kinetics of adsorption when using our protocols. Therefore we chose to follow the shift of the peak of the plasmon resonance. As the plasmon resonance peak is sensitive to refractive index changes on the surface, we expect the kinetics of the change to approximate the kinetics of the DNA attachment to the surface. As can be seen in Figure 2A, even when using a high-end spectrophotometer to scan the extinction of the nanoparticles around the plasmon peak with a resolution of $0.2 \mathrm{~nm}$, using the point of highest absorbance on the graph gives rise to very high noise. One can quantify the RMS noise of the repeated measurement of unmodified particles and reach a value of $0.28 \mathrm{~nm}$. However, if instead of taking the point of highest absorbance, we fit the full spectrum data (200 data points $-500 \mathrm{~nm}$ to $540 \mathrm{~nm}$, at $0.2 \mathrm{~nm}$ resolution) to a lorenzian using MATLAB and then extract the exact position of the peak, we get a much smoother curve, with a very low noise level (RMS noise of $0.0046 \mathrm{~nm}$ ) which is accurate enough to discern the kinetics of the binding, Figure 2B.

The kinetics can be fitted $\left(\mathrm{R}^{2}=0.99987\right)$ to a double exponent. The origin of this double exponent can be explained by an initial fast adsorption of molecules in a random orientation on the surface of the particles, followed by a slower process of the creation of a dense highly ordered layer. 


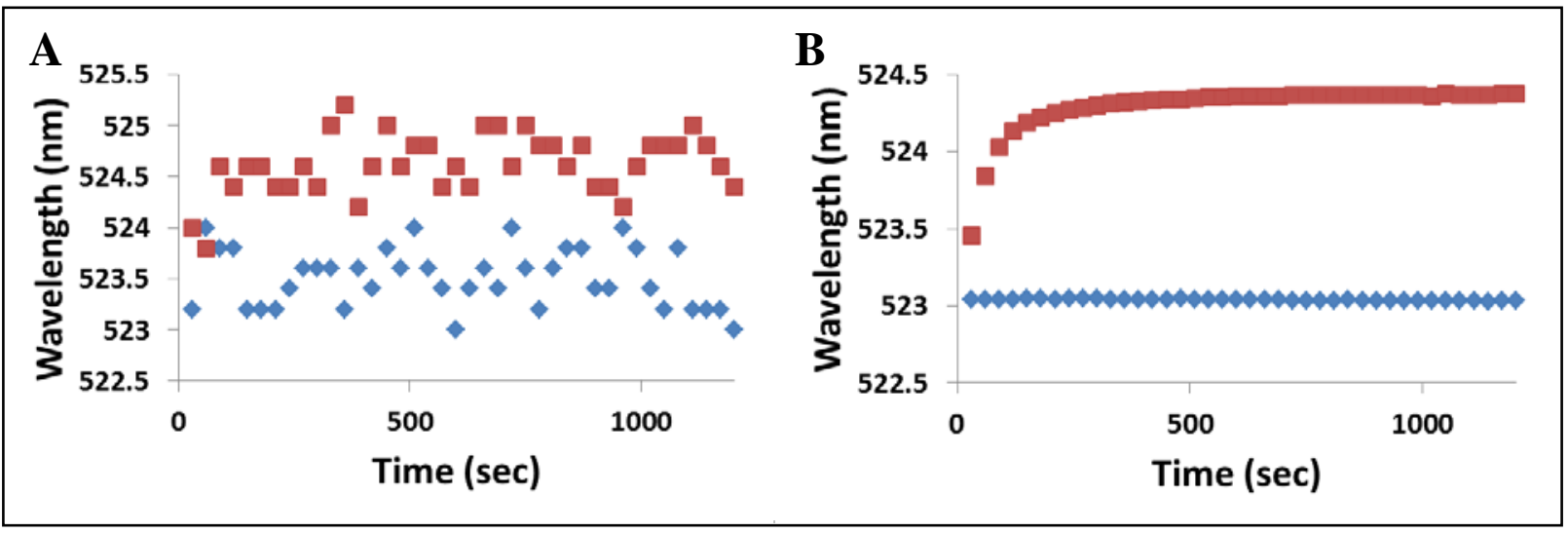

Figure. 2: Time dependence of the position of the plasmon absorbance peak of the nanoparticles as determined by (A) the point of highest absorbance and (B) The peak of a fitted curved created by fitting the full absorbance peak to a Lorenzian. In both cases the blue points are for the gold nanoparticles before modification, and the red points are after the addition of $1 \mu \mathrm{l}$ of $100 \mu \mathrm{M}$ ZNA. Absorption scans between $500 \mathrm{~nm}$ and $540 \mathrm{~nm}$ with a resolution of $0.2 \mathrm{~nm}$ were measured every 30 sec for 20 minutes.

Using Lorenzian fitting, we can now compare the kinetics of the two modification methods. It should be noted that when adding the citrate buffer at $\mathrm{pH}=3$, there is a jump in the signal which is related to a bulk refractive index(RI) change, rather than a local change of the refractive index at the surface of the particles. We quantified this change using unmodified nanoparticles in order to compare the two kinetic curves. Figure 3 shows the obtained time-dependent curves for modification with ZNA and modification with DNA in the acidic citrate method. Two major differences are noticeable. First, the adsorption kinetics of ZNA is much higher than that of DNA at $\mathrm{pH}=3$. Second, if we assume that the RI change per DNA strand is about the same in both cases (in practice the MW of the ZNA is about 20\% less than that of the DNA used), we can see that the total of DNA bound to the nanoparticle is almost 2x lower than for ZNA, even in the case of long incubation times. Therefore, simply increasing the incubation time will not bring about stable modification of concentrated gold particles using the acidic citrate method (see Figure 1A (iv)). The fact that both the kinetics and the capacity (maximum loading of DNA strands) on the nanoparticles are lower can be explained by the higher negative charge of the DNA, even at $\mathrm{pH}=3$, compared to ZNA, which not only causes a lower binding rate to the negatively charged particle surface, but also increases electrostatic repulsion between DNA strands on the surface of the nanoparticle.

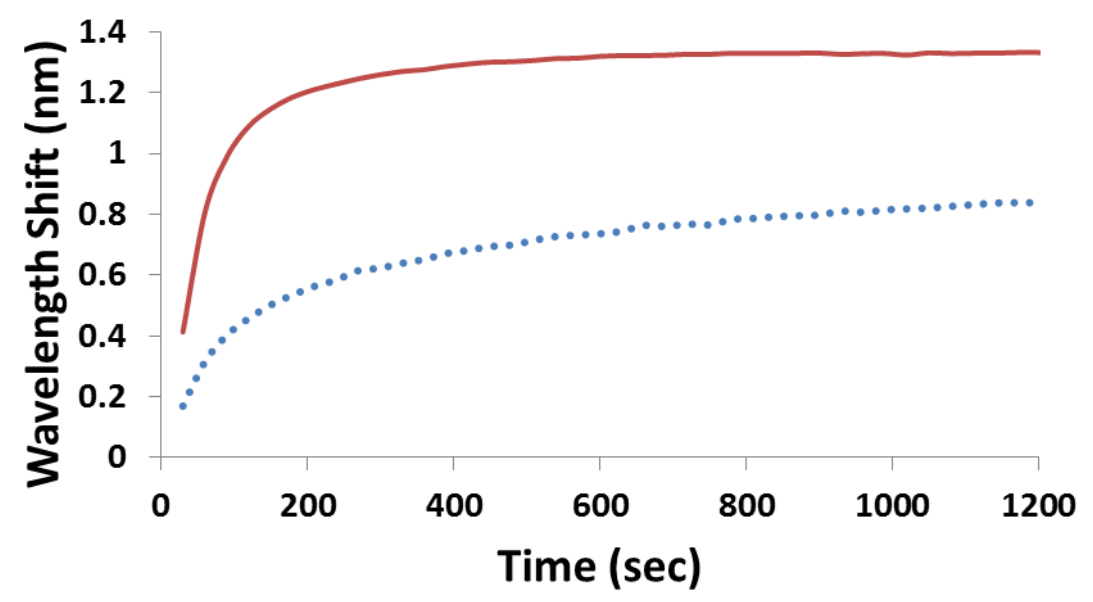

Figure. 3: Time dependence of the position of the plasmon absorbance peak of the gold nanoparticles when using DNA with the acidic citrate method (dotted line) or ZNA (full line). 


\subsection{Modification of silver nanoparticles}

The same procedure that was used for the modification of gold nanoparticles with ZNA, could also be used to modify silver nanoparticles. In general, silver nanoparticles are considered more difficult to modify. This stems from two main reasons: one is that the silver-thiol bond is weaker than the gold-thiol bond, the other is that silver is less stable than gold and therefore tends to oxidize during the long incubation times usually used so far for DNA modification of nanoparticles. However as the ZNA modification is very fast and is not based on thiol chemistry, we found that silver nanoparticles can be effectively modified with ZNA-based DNA as easily as gold nanoparticles. As can be seen in Figure 4, after modification and the addition of salt, we only get a small peak shift which is related to the modification of the surface with high MW species, without any secondary aggregation peak in the 500-660 nm range.

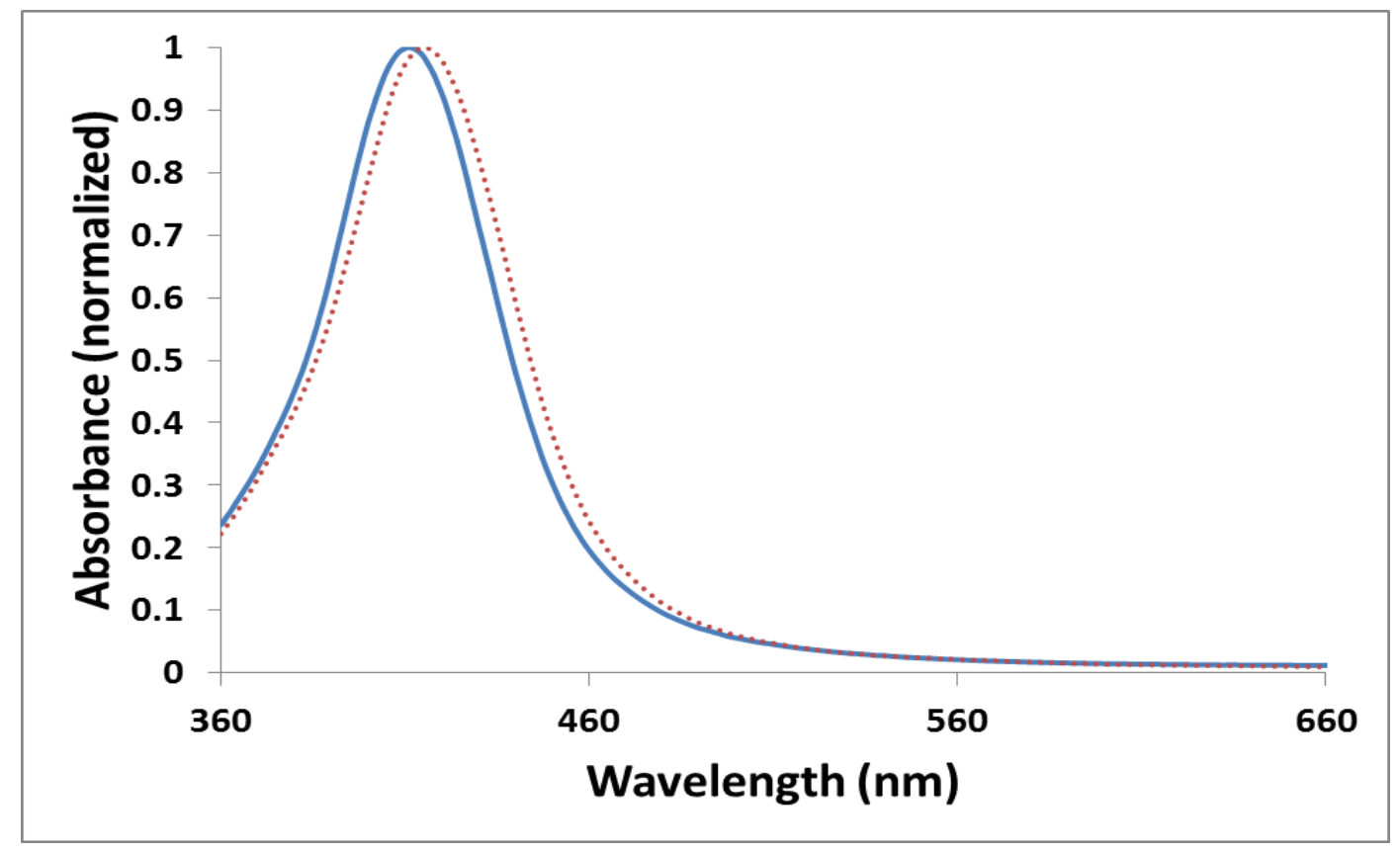

Figure. 4: Absorbance scan of silver nanoparticles before (full line) and after modification with ZNA and the addition of salt (dotted line).

\section{CONCLUSIONS}

Fast and efficient modification of large gold nanoparticles can be achieved when the total charge of the DNA is decreased. Although modifying particles at $\mathrm{pH}=3$ reduces the charge of the DNA and can give fast and efficient modification of smaller gold nanoparticles, this method cannot be used for larger particles, unless they are highly concentrated. Here we demonstrate, that by using ZNA (DNA with a polycationic tail), fast modification of large particles can occur, as the total charge on the DNA is even lower in the case of ZNA as compared to DNA at pH=3. Using time-dependent absorbance measurements, we observe both faster kinetics of adsorption of ZNA, and also a greater total shift in the plasmon resonance for ZNA, suggesting that the DNA/particle ratio for ZNA is higher than for DNA. Lastly, we show that this method is also effective for the modification of silver nanoparticles, which are usually more prone to aggregation during the long incubation times of previously used methods.

\section{ACKNOWLEDGEMNTS}

Part of the work of R.G. was funded by an NWO Veni grant (No. 700.10.410). The work of K. G. was funded by the Netherlands Technology Foundation STW (STW-project no. 11818). 


\section{REFERENCES}

[1] Saha, K., Agasti, S. S., Kim, C., Li, X. and Rotello, V. M., "Gold Nanoparticles in Chemical and Biological Sensing," Chem. Rev. 112(5), 2739-2779 (2012).

[2] Verdoold, R., Gill, R., Ungureanu, F., Molenaar, R. and Kooyman, R. P. H., "Femtomolar DNA detection by parallel colorimetric darkfield microscopy of functionalized gold nanoparticles," Biosensors and Bioelectronics 27(1), 77-81 (2011).

[3] Tong, L., Wei, Q., Wei, A. and Cheng, J.-X., "Gold Nanorods as Contrast Agents for Biological Imaging: Optical Properties, Surface Conjugation and Photothermal Effectst," Photochem. Photobiol. 85(1), 21-32 (2009).

[4] Murphy, C. J., Gole, A. M., Stone, J. W., Sisco, P. N., Alkilany, A. M., Goldsmith, E. C. and Baxter, S. C., "Gold Nanoparticles in Biology: Beyond Toxicity to Cellular Imaging," Acc. Chem. Res. 41(12), 1721-1730 (2008).

[5] Geoghegan, W. D. and Ackerman, G. A., "Adsorption of horseradish peroxidase, ovomucoid and antiimmunoglobulin to colloidal gold for the indirect detection of concanavalin A, wheat germ agglutinin and goat anti-human immunoglobulin $\mathrm{G}$ on cell surfaces at the electron microscopic level: a new method, theory and application," J. Histochem. Cytochem. 25(11), 1187-1200 (1977).

[6] Mirkin, C. A., Letsinger, R. L., Mucic, R. C. and Storhoff, J. J., "A DNA-based method for rationally assembling nanoparticles into macroscopic materials," Nature 382(6592), 607-609 (1996).

[7] Elghanian, R., Storhoff, J. J., Mucic, R. C., Letsinger, R. L. and Mirkin, C. A., "Selective colorimetric detection of polynucleotides based on the distance-dependent optical properties of gold nanoparticles," Science 277(5329), 1078-1081 (1997).

[8] Kanaras, A. G., Wang, Z., Bates, A. D., Cosstick, R. and Brust, M., "Towards Multistep Nanostructure Synthesis: Programmed Enzymatic Self-Assembly of DNA/Gold Systems," Angewandte Chemie International Edition 42(2), 191-194 (2003).

[9] Stoeva, S. I., Lee, J.-S., Thaxton, C. S. and Mirkin, C. A., "Multiplexed DNA Detection with Biobarcoded Nanoparticle Probes," Angewandte Chemie International Edition 45(20), 3303-3306 (2006).

[10] Zu, Y. and Gao, Z., "Facile and Controllable Loading of Single-Stranded DNA on Gold Nanoparticles," Anal. Chem. 81(20), 8523-8528 (2009).

[11] Zhang, X., Servos, M. R. and Liu, J., "Instantaneous and Quantitative Functionalization of Gold Nanoparticles with Thiolated DNA Using a pH-Assisted and Surfactant-Free Route," J. Am. Chem. Soc. 134(17), 7266-7269 (2012).

[12] Zhang, X., Gouriye, T., Göeken, K., Servos, M. R., Gill, R. and Liu, J., "Toward Fast and Quantitative Modification of Large Gold Nanoparticles by Thiolated DNA: Scaling of Nanoscale Forces, Kinetics, and the Need for Thiol Reduction," The Journal of Physical Chemistry C 117(30), 15677-15684 (2013).

[13] Gill, R., Goeken, K. and Subramaniam, V., "Fast, single-step, and surfactant-free oligonucleotide modification of gold nanoparticles using DNA with a positively charged tail," Chem. Commun. 49(97), 11400-11402 (2013).

[14] Moreau, V., Voirin, E., Paris, C., Kotera, M., Nothisen, M., Rémy, J.-S., Behr, J.-P., Erbacher, P. and LenneSamuel, N., "Zip Nucleic Acids: new high affinity oligonucleotides as potent primers for PCR and reverse transcription," Nucleic Acids Res. 37(19), e130 (2009).

[15] Karpovich, D. S. and Blanchard, G. J., "Direct Measurement of the Adsorption Kinetics of Alkanethiolate SelfAssembled Monolayers on a Microcrystalline Gold Surface," Langmuir 10(9), 3315-3322 (1994). 